\title{
LA THÉORIE DU BALLAST
}

\author{
Par
}

\section{le Professeur-Docteur Franz LEHMANN}

(Göttingen)

Zeitschrift für Tierernährung und Futtermittelkunde

Band 5, I94I, pages $155^{-1} 73$

Traduit par André VOISnN

La notion de ballast n'est pas mentionnée dans les ouvrages anciens sur l'alimentation des animaux. Elle n'apparaît que récemment dans les manuels et les tables d'alimentation des animaux, sans cependant être étudiée et expliquée de plus près. De plus, il semble que cette notion n'est pas toujours considérée avec la prudence voulue ; aussi, me paraît-il nécessaire de bien voir son importance et de déterminer son origine. Je serai malheureusement pour cela, obligé de parler de ma propre personne, car c'est moi-même qui, au cours de mes conférences à Göttingen sur la théorie de 1'alimentation des animaux, ai fait ressortir pour la première fois la notion fondamentale de ballast.

J'appelle "ballast " la substance organique non digestible de l'aliment. Il se calcule en additionnant les parties digestibles: Albumines, matières grasses, fibres cellulosiques ( $\left.{ }^{1}\right)$ et matières extractives non azotées, puis on déduit ce total de la matière organique trouvée par analyse.

La conception de ballast a son origine dans des considérations pédagogiques. Au cours des conférences où on doit parler des normes des aliments fourragers, le chiffre "matière sèche " est celui le moins facile à définir. Je cherchais donc une simplification. Elle apparut d'elle-même, quand, au lieu de la " matière sèche ", je pris comme point de départ de mes explications, la "substance organique totale ". Celle-ci peut facilement être mise en parallèle avec la " substance organique digestible ".

Il s'y ajouta alors qu'Emil WoLFF qui, pendant des dizaines d'années, calcula presque seul toutes les normes fourragères, indiquait toujours dans ses tables : "substance organique totale " et non la matière sèche. Je me suis donc reporté aux tables de WoLFF et en déduisis d'abord le petit tableau suivant :

\footnotetext{
(1) Le terme "fibres cellulosiques " correspond en général au mot fuançais " cellulose ". Dans le texte, nous avons cru cependant préférable de laisser le mot "fibres " tout court.
} 
TABLEAU I

\begin{tabular}{|c|c|c|c|}
\hline \multirow[b]{2}{*}{$\begin{array}{l}\text { Pour un animal de } 500 \mathrm{~kg} \\
\text { de poids vif } \\
\text { du type sujvant : }\end{array}$} & \multicolumn{3}{|c|}{ Normes nécessaires pour ces animaux } \\
\hline & $\begin{array}{c}\text { substance } \\
\text { organique } \\
\text { totale } \\
\mathrm{kg}\end{array}$ & $\begin{array}{c}\text { substance } \\
\text { organique } \\
\text { digestible } \\
\mathrm{kg}\end{array}$ & $\begin{array}{c}\text { substance } \\
\text { organique } \\
\text { non digestible } \\
\text { (ballast) } \\
\mathrm{kg}\end{array}$ \\
\hline $\begin{array}{l}\text { Bovins tranquilles à l'étable } \ldots \ldots \ldots \ldots \\
\text { Vaches laitières (en laiti.................. } \\
\text { Boeufs à l'engrais (I re période) } \ldots \ldots \ldots\end{array}$ & $\begin{array}{r}8,8 \\
12,0 \\
13,5\end{array}$ & $\begin{array}{l}4,5 \\
7,7 \\
9,0\end{array}$ & $\begin{array}{l}4,3 \\
4,3 \\
4,5\end{array}$ \\
\hline
\end{tabular}

Ainsi, la ration d'entretien et la ration de production donnent un chiffre identique pour la matière organique non digestible. Ceci était évidemment surprenant, mais l'explication n'était pas difficile.

Je suis, comme Emil WoLFF, un représentant des premiers temps de la theorie de l'alimentation du bétail ; et à cette époque, nous étions en liaison étroite avec le paysan et ses méthodes de travail. En ce temps-là, les stations expérimentales se trouvaient dans les fermes mêmes, et les normes fourragères étaient déduites à partir des méthodes d'alimentation paysannes. Les chiffres de nos premières tables d'alimentation étaient en réalité déduits des rations réelles que des cultivateurs avertis donnaient à leurs animaux.

Quand il s'agit de fournir à un bouf sa seule ration d'entretien, le cultivateur essaie de le nourrir le plus économiquement possible, c'est-à-dire avec de la paille, des menues-pailles et du fourrage, tout en lui donnant le moins possible d'aliments à forte digestibilité, qui sont toujours chers. Or, tous les aliments bon marché, courants à la ferme, sont des aliments riches en ballast. Les nourritures données pour l'entretien seul contiendront donc autant de ballast que l'animal peut absorber, et ceci apparaît dans le chiffre de $4,3 \mathrm{~kg}$ de ballast pour un animal d'un poids de $500 \mathrm{~kg}$.

Mais, si on veut qu' 'un bœuf prenne de la viande ou qu'une vache donne du lait, il faut leur fournir une beaucoup plus grande quantité de matières digestibles. Seule, la quantité de ballast ne peut être angmentée, car avec $4,3 \mathrm{~kg}$ de ballast, la capacité d'absorption de ballast de l'animal a déjà atteint sa limite. C'est ce qui explique que dans mon petit tableau I, les normes de la ration pour la vache en lait contiennent exactement la même quantité de substance organique non digestible que la ration d'entretien, à savoir $4,3 \mathrm{~kg}$. La ration du bœuf à l'engrais contient $4,5 \mathrm{~kg}$ de substance organique non digestible, chiffre qui, par conséquent, est encore peu éloigné du chiffre de la ration d'entretien.

Il est intéressant de noter qu'Emil WoLfF divise l'engraissement qui dure généralement 3 à 4 mois en trois périodes. Nous avons toujours eu difficile à comprendre cette division. En effet, Emil WoLFF indique les matières nutritives 
suivantes (tableau II) comme nécessaires pour les bœufs de $500 \mathrm{~kg}$ au cours de ces trois périodes :

\section{TABLEAU II}

Besoins des bouts de $500 \mathrm{~kg}$ à l'engrais

\begin{tabular}{|c|c|c|c|}
\hline r & $\begin{array}{l}\text { Albumine } \\
\text { digestible } \\
\mathrm{kg}\end{array}$ & $\begin{array}{l}\text { Hydrocarbones } \\
\text { kg }\end{array}$ & $\begin{array}{l}\text { Matières } \\
\text { grasses } \\
\mathrm{kg}\end{array}$ \\
\hline 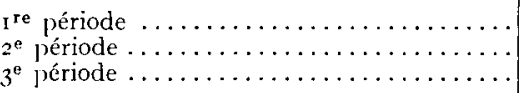 & $\begin{array}{l}1,25 \\
1,50 \\
1,35\end{array}$ & $\begin{array}{l}7,5 \\
7,4 \\
7,4\end{array}$ & $\begin{array}{l}0,25 \\
0,35 \\
0,30\end{array}$ \\
\hline
\end{tabular}

Ces chiffres sont au premier abord incompréhensibles, à moins d'en étudier l'origine : l'éminent et remarquable savant EMIL WOLFF les avait déduits de ses expériences pratiques basées sur les méthodes usuelles d'engraissement des cultivateurs. Or, à cette époque, les cultivateurs avertis divisaient leur engraissement en trois périodes. Du reste, d'autres spécialistes de l'alimentation de cette époque ont fait de même ; c'est le cas de SETTEGAST dans son ouvrage sur l'alimentation qui fut publié en 1872 .

L'explication m'apparut clairement quand je calculais les rations de WoLFF en "substance organique totale " et en déduisis la substance organique digestible, ce qui me donna le ballast. Le résultat de mon calcul est donné dans le tableau III :

\section{TABLEAU III}

Besoins des boufs de $500 \mathrm{~kg}$ à l'engrais (corollaire du tableau II)

\begin{tabular}{|c|c|c|c|}
\hline & $\begin{array}{c}\text { Substance } \\
\text { organique totale } \\
\text { les }\end{array}$ & $\begin{array}{l}\text { Substance } \\
\text { orgenique } \\
\text { digestible } \\
\qquad k g r\end{array}$ & $\begin{array}{l}\text { Substance orga- } \\
\text { nique non } \\
\text { digestible (ballast) } \\
\mathrm{kg}\end{array}$ \\
\hline 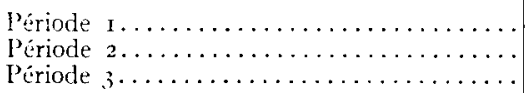 & $\begin{array}{l}13,5 \\
13,0 \\
12,5\end{array}$ & $\begin{array}{l}9,00 \\
9,25 \\
9,05\end{array}$ & $\begin{array}{l}4.50 \\
3.75 \\
3,45\end{array}$ \\
\hline
\end{tabular}

La quantité de ballast, au cours de ces trois périodes, décroît de $4,5 \mathrm{~kg}$ à $3,75 \mathrm{~kg}$ puis $3,45 \mathrm{~kg}$. Car, au fur et à mesure que l'engraissement progresse et que les cavités de l'abdomen se remplissent de graisse, la capacité d'absorption de ballast devient plus faible. Par conséquent, si on veut finir parfaitement l'engraissement, c'est-à-dire maintenir jusqu'à la fin un accroissement quotidien constant de poids, il faut donner au bœuf un aliment plus pauvre en ballast, sans cependant diminuer la quantité de substances organiques digestibles.

Aujourd'hui, on ne parle plus de diviser l'engraissement en trois périodes ; 
ce n'est du reste pas nécessaire, car au fur et à mesure que l'engraissement progresse, les bêtes veillent elles-mêmes à manger moins de paille.

Du reste, nos calculs ont leurs limites : la quantité de ballast absorbé n'est pas un chiffre constant ; elle décroît quand l'engraissement avance. Nous trouverons en outre d'autres facteurs de variations de ce chiffre fondamental.

\section{Le ballast dans l'alimentation des vaches laitières}

L'ancienne norme pour une vache de $500 \mathrm{~kg}$, si on néglige les chiffres pour chacun des éléments nutritifs individuels, était la suivante :

\begin{tabular}{|c|c|c|c|}
\hline & $\begin{array}{l}\text { Substance } \\
\text { organique } \\
\text { totale } \\
\text { kg }\end{array}$ & $\begin{array}{c}\text { Substance } \\
\text { organiqu } \\
\text { digestible } \\
\text { kg }\end{array}$ & $\begin{array}{l}\text { Pallast } \\
\qquad k g\end{array}$ \\
\hline 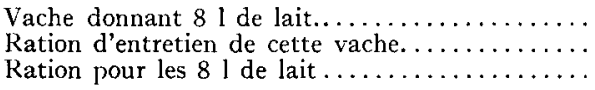 & $\begin{array}{r}12,0 \\
8,8 \\
3,2\end{array}$ & $\begin{array}{l}7,7 \\
4,5 \\
3,2\end{array}$ & $\begin{array}{l}+, 3 \\
4.3 \\
0\end{array}$ \\
\hline
\end{tabular}

On pourrait être tenté de calculer la substance organique nécessaire pour produire $\mathrm{r}$ litre de lait, et d'utiliser ces normes pour calculer les rations nécessaires aux vaches très fortes productrices. Nous trouvons alors qu'il faut par litre de lait $0,4 \mathrm{~kg}$ de substance organique digestible, et on pourrait alors dresser une table où on indiquerait les besoins de la vache suivant sa production de lait ; c'est ce que j'ai fait et que 1'on a fait depuis mes conférences à 1'Université de Göttingen en 1895 .

Comme la quantité de ballast demeure constante et égale à $4,3 \mathrm{~kg}$, il n'y a que la quantité absorbée de substance organique digestible, à savoir $4,0 \mathrm{~kg}$ par Io litre de lait qui augmente. La quantité d'aliments fournis doit donc permettre de satisfaire cette augmentation de besoins en substance organique digestible. Ceci apparaît très nettement quand on calcule le coefficient de digestibilité de la substance organique de l'aliment fourni, comme nous l'avons fait dans le tableau IV.

\section{TABI,EAU IV}

Besoins de la vache suivant sa production de lait

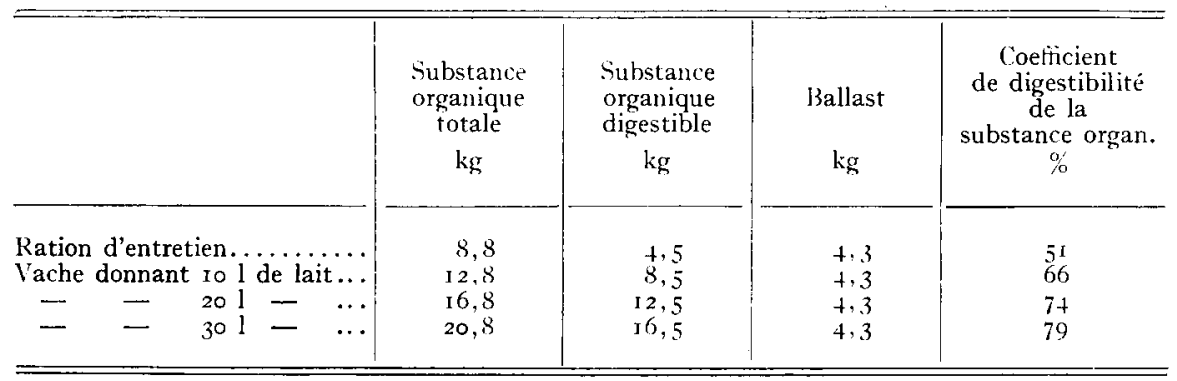


Ce tableau est très instructif, surtout si on compare les coefficients de digestibilité du' tableau avec ceux d'aliments courants : celui du fourrage est de 6 I $\%$ et de la paille $40 \%$.

Pour fournir à un bovin sa ration d'entretien, il suffit d'un mélange de fourrage et de paille avec un coefficient de digestibilité de $5 \mathrm{I} \%$.

Ia vache qui donne io litres de lait ne peut déjà plus satisfaire ses besoins avec du fourrage, mais doit recevoir un aliment avec un coefficient de digestibilité plus élevé.

Dans le cas de la vache donnant 20 litres de lait, le coefficient de digestibilité de l'aliment fourni doit être de $74 \%$ c'est-à-dire plus élevé que celui du son, pour lequel le coefficient est de $72 \%$. Pour les vaches qui donnent 30 litres de lait, il faut un fourrage avec un coefficient de $79 \%$. Rappelons que les provendes de céréales ont un coefficient de digestibilité de $8 \mathrm{r}-82 \%$.

Quand la quantité de lait produit augmente, la vache exige non seulement un aliment plus riche en matières nutritives, mais elle exige également une autre espèce d'aliments. Il faut donner de moins en moins de fourrage et de paille. Une alimentation rationnelle des grandes laitières, c'est-à-dire une alimentation qui ne fasse pas perdre du poids à la vache, exige des quantités énormes d'aliments concentrés.

Ce principe, qui est facilement démontré par le calcul du ballast, est aujourd'hui parfaitement connu de tous les agriculteurs. Mais, maintenant, on calcule différemment, grâce aux travaux de KEI,INER, les besoins en éléments nutritifs. On résume le besoin global en éléments nutritifs dans un seul chiffre, la "valeur amidon ", et on indique en même temps le besoin en albumine digestible.

Comme besoin en substances nutritives, pour ro litres de lait, je prends les chiffres suivants :

Unités amidon ........................ $2,5 \mathrm{~kg}$

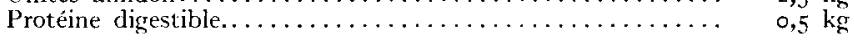

J'en déduis le tableau $\mathrm{V}$.

TABLEAU V

Besoins en éléments nutritifs d'une vache suivant sa production de lait

\begin{tabular}{ll|l|r|r}
\hline & & \\
\end{tabular}


D'après ces chiffres de base, nous pouvons déterminer les mélanges d'aliments permettant de satisfaire à ces besoins. Comme aliment grossier volumineux, on utilise la paille et le fourrage, que nous complétons par un mélange de tourteaux. Nous fournissons en outre, des pulpes et des pommes de terre séchées ainsi que du son de blé. Le tableau VI vous indique les rations :

\section{TABleau VI}

Rations pour vaches de différentes productions

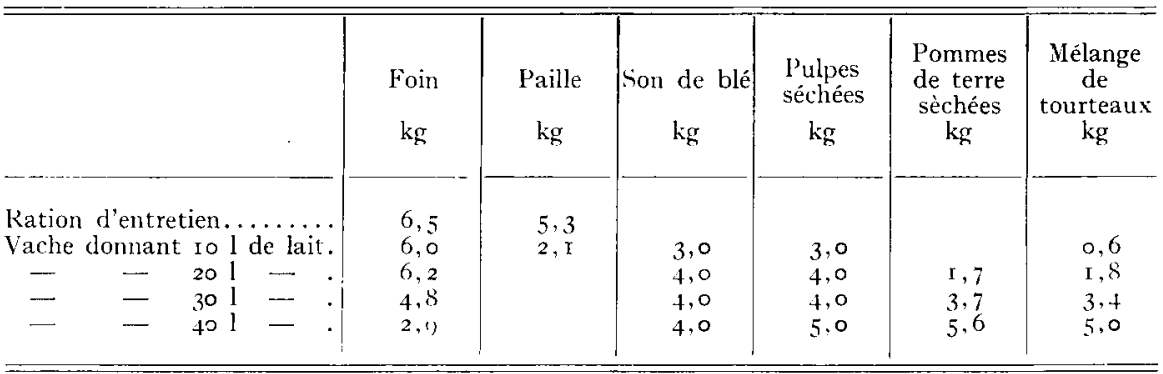

․ B. - Voir caractéristiques de ces aliments au tableau VII.

L'attention que nous avons apportée à ne pas dépasser la quantité maxima de ballast se révèle par la suppression de la paille au-dessus de io litres de lait et par la diminution progressive de la quantité de fourrage. Pour la ration d'entretien, le fourrage et la paille suffisent. Mais, déjà, avec ıo litres de lait, il faut beaucoup réduire la quantité de paille. Pour les vaches avec 30 litres de lait, la quantité de fourrage doit de plus être très restreinte. Le calcul pour une vache de 40 litres est déjà d'une certitude relative; il est douteux qu'une vache donnant 40 litres de lait puisse absorber la ration contenant $3 \mathrm{~kg}$ de fourrage.

\section{Le ballast des aliments}

Les calculs ne peuvent être faits de manière précise que si nous étudions de plus près les richesses en éléments nutritifs de nos principaux aliments fourragers. De plus, nous devons, pour ces aliments, indiquer le ballast en même temps que leur richesse en unités amidon et en albumine digestible. Ces chiffres se déduisent facilement des tables de KELLNER. Je les ai indiqués pendant des dizaines d'années aux élèves de mes cours, mais ne les ai jamais publiés. Malgré cela, mes chiffres ont été utilisés dans de nombreuses tables. J'indique dans le tableau VII les richesses des éléments employés dans le tableau VI.

Le mélange de tourteaux était composé comme suit :

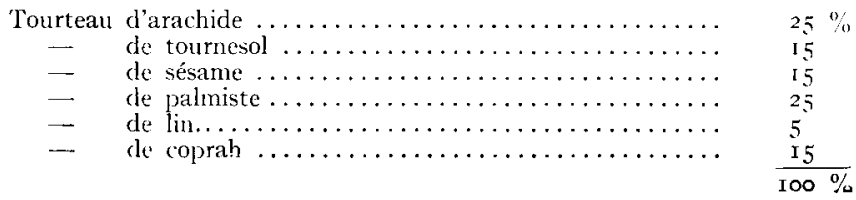




\section{TABLEAU VII}

Caractéristiques de quelques aliments fourragers

\begin{tabular}{|c|c|c|c|}
\hline & \multicolumn{3}{|c|}{ Pour 1 ooo g d'aliment, on a : } \\
\hline & $\begin{array}{l}\text { Albumine } \\
\text { digestible } \\
\mathrm{g}\end{array}$ & $\begin{array}{c}\text { Valeur amidon } \\
\mathrm{g}\end{array}$ & $\begin{array}{c}\text { Ballast } \\
\mathrm{g}\end{array}$ \\
\hline Mélange de tourteaux ............. & 285 & 742 & 1.79 \\
\hline Foin de prairie (qualité moyenne) ..... & 38 & 3 Io & 328 \\
\hline 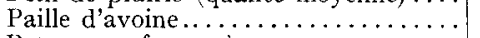 & IO & 170 & 408 \\
\hline Betteraves fourragères............. & I & 63 & 15 \\
\hline 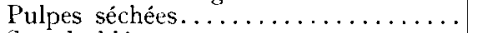 & 36 & 519 & 176 \\
\hline Son de blé. $\ldots \ldots \ldots \ldots \ldots \ldots \ldots \ldots$ & gI & 426 & 260 \\
\hline Pommes de terre séchées........... & 36 & 737 & 90 \\
\hline
\end{tabular}

\section{La « Proportion de ballast " (Ballastverhältnis)}

L'introduction de la notion de ballast complique indubitablement le calcul des rations.

Il n'est pas facile d'introduire ce nouvel élément dans nos tables habituelles ce qui nous oblige à tenir compte de trois éléments au lieu des deux éléments qui s'y trouvent. Aussi, est-il nécessaire de simplifier le plus possible nos calculs ; pour cela, il est nécessaire qu'on puisse juger rapidement de la teneur en ballast, qu'elle soit forte ou faible. La comparaison est assez facile quand il s'agit d'aliments secs ; mais devient difficile avec des aliments riches en eau tels que des fourrages verts ou des betteraves fourragères. Aussi, pour faciliter les calculs, recommandons-nous l'introduction de la "proportion de ballast" (Ballastverhältnis), qui indique combien l'aliment contient de ballast pour Ioo unités amidon. Ce chiffre s'est révélé dans la pratique fort précieux, et je l'indique depuis quelques années dans mes tables.

A titre d'exemple, je donne dans le tableau VIII, cette proportion pour les aliments portés dans le tableau VII.

\section{TABLEAU VIII}

La "proportion de ballast" de quelques aliments fourragers

\begin{tabular}{|c|c|c|c|}
\hline & \multicolumn{3}{|c|}{ Pour I ooo g d'aliment, on a : } \\
\hline & $\begin{array}{c}\text { Valeur amidon } \\
A \\
g\end{array}$ & $\begin{array}{l}\text { Ballast } \\
\qquad \begin{array}{c}B \\
8\end{array}\end{array}$ & $\begin{array}{l}\text { Proportion } \\
\text { de ballast } \\
\text { roo } \times \frac{\mathrm{B}}{\mathrm{A}}\end{array}$ \\
\hline 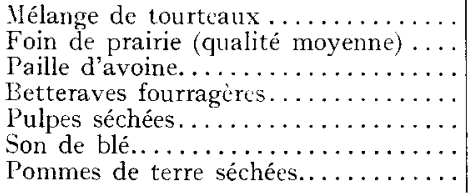 & $\begin{array}{r}74,2 \\
31,0 \\
17,0 \\
6,3 \\
51,0 \\
42,6 \\
73,7\end{array}$ & $\begin{array}{r}17,9 \\
32,8 \\
40,8 \\
1,5 \\
17,6 \\
26,9 \\
9,0\end{array}$ & $\begin{array}{r}24 \\
106 \\
240 \\
24 \\
34 \\
63 \\
12\end{array}$ \\
\hline
\end{tabular}




\section{La quantité de ballast absorbée est-elle une constante ?}

La théorie du ballast s'appuie sur le principe suivant : chez les bêtes adultes, la quantité de ballast absorbée est constante. Comme le ballast représente la partie non digestible des substances organiques absorbées, le principe peut encore s'exprimer de la manière suivante : la quantité de substance organique contenue quotidiennement dans la fiente est une constante dans le cas d'une alimentation rationnelle. Il en résulte donc que ce principe doit être facilement vérifié par des essais pratiques.

Or, nous avons déjà constaté une exception à ce principe : c'est dans le cas de bœufs à l'engraissement qui étaient pourtant des bêtes adultes ; en effet, quand 1'engraissement avance, la possibilité d'absorption de ballast par l'animal diminue.

Dans le cas des vaches, il semble que ce phénomène ne se produit pas. En effet, les aliments fournis et calculés d'après la production de lait ne couvrent que les besoins de la vache sans l'engraisser et par conséquent, sans amener un remplissage des cavités de l'abdomen. Le chiffre de $4,3 \mathrm{~kg}$ de ballast absorbé serait cependant mal compris, si on le généralisait. I1 n'est pas valable pour tous les bovins, mais est différent suivant les individus. Il dépend probablement de la manière dont 1'animal a été élevé, mais il demeure constant pour un animal donné quelle que soit la nature des aliments fournis. Nous pouvons également affirmer avec confiance que le chiffre du ballast, à part ces variations particulières, est une constante. Nous pouvons pour cette affirmation nous appuyer sur les chiffres d'Emil WoLFF qui les avait déduits de multiples observations.

Par contre, les nombreuses recherches théoriques et pratiques, qui ont été entreprises depuis, ne confirment pas le chiffre élevé de ballast absorbé que nous indiquons. On peut constater que ces recherches indiquent des chiffres de ballast absorbé plus faibles, et ceci est compréhensible. Des essais précis d'alimentation supposent que les aliments fournis seront absorbés suivant certaines règles quantitatives; or, cette absorption quantitative précise ne peut se faire que si le total du ballast contenu dans les aliments absorbés est au-dessous de la quantité limite de ballast absorbable. Ceci est en particulier le cas dans les expériences de digestibilité et de métabolisme. Mais de telles expériences ne correspondent pas aux conditions d'alimentation réelles, telles qu'elles se présentent à l'étable. Dans ce cas, les aliments grossiers volumineux, plus exactement la paille et la menue-paille, ne sont jamais complètement absorbés par l'animal. On présente toujours à l'animal beaucoup plus de paille qu'il n'en mangera parce que les bovins, et les moutons peuvent ainsi choisir et manger la partie la meilleure. Or, ce qui rend douteux et difficiles nos essais scientifiques d'alimentation, c'est qu'ils s'éloignent des méthodes d'alimentation pratique bon marché, utilisées par nos agriculteurs. 
D'autre part, quand on étudie les rations réelles que donnent nos cultivateurs aux vaches à l'étable, on trouve d'énormes différences suivant la méthode de nourriture dans les différentes fermes, que ce soit une nourriture économique à base de paille ou une nourriture riche en aliments à digestibilité élevée.

Je connais par exemple un cas, où des vaches de $500 \mathrm{~kg}$ donnaient I2 litres de lait, en recevant une ration contenant $8,8 \mathrm{~kg}$ de paille avec I $522 \mathrm{~g}$ d'unitésamidon. Cette quantité de paille pouvait être mangée par la vache, parce que celle-ci possédait la capacité d'absorber une quantité de ballast beaucoup plus élevée que les $4,3 \mathrm{~kg}$ théoriques. Pour simplifier, supposons que la vache produisait Io litres de lait, de manière à pouvoir faire une comparaison avec nos normes précédemment indiquées. D'après ces normes, nous avons besoin pour Io litres de lait de 5500 unités amidon, soit pour :

$$
\text { I } 1 \text { de lait ...................... } 550 \text { unites anidon }
$$

Les mêmes normes exigent :

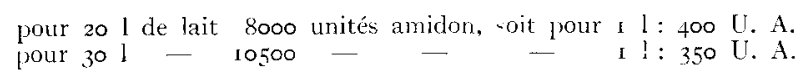

Il en résulte que plus la production augmente moins les aliments fournis ont besoin d'apporter d'éléments nutritifs par litre de lait. C'est du reste pour cela que l'on s'efforce de sélectionner des vaches à grande capacité laitière.

Notre cultivateur avait suivi une autre voie. Il se contentait de rendements plus faibles en lait et avait choisi des vaches qui avec ce rendement faible, étaient cependant capables d'absorber $8,8 \mathrm{~kg}$ de paille. Dans cette paille se trouvent I $5^{22} \mathrm{~g}$ d'unités-amidon qui pratiquement ne lui coûtent rien, car il les aurait utilisés normalement comme litière. Les Io litres de lait exigeaient comme matières nutritives payées la différence :

$$
5500 \mathrm{~g}-\mathrm{I} 522 \mathrm{~g}=3978 \mathrm{~g} \text { d'unités-amidon, }
$$

autrement dit, pour un litre de lait, on dépensait $398 \mathrm{~g}$ d'unités-amidon, alors que nous venons de voir qu'avec l'absorption normale de $4,3 \mathrm{~kg}$ de ballast et une production de 20 litres de lait, nous avions besoin pour un litre de lait de $400 \mathrm{~g}$ d'unités-amidon.

Or, nous avons vu dans le tableau VI que pour obtenir 20 litres de lait, avec l'absorption normale de $4,3 \mathrm{~kg}$ de ballast, il n'était pas possible de donner de la paille dans la ration. Il y a donc une autre manière de réduire le prix de revient du lait. Malgré toute la propagande pour la sélection et l'entretien de vaches à haut rendement, les petits cultivateurs, qui forment la majorité en Allemagne centrale et métidionale, s'obstinent à nourrir leurs vaches de la manière dont nous venons de parler, sans se préoccuper du faible rendement en lait qu'ils obtiennent, parce qu'ils préfèrent éviter d'acheter des aliments concentrés coûteux et aiment mieux utiliser les aliments produits à la ferme. Le calcul grossier que nous venons de faire, explique donc une théorie du ballast qui est appliquée chez nos paysans depuis les temps les plus reculés. 
La théorie de ballast amène à apprécier les aliments concentrés (y compris les betteraves et les pommes de terre) suivant le principe suivant: plus un aliment est digestible ou inversement plus est faible la " proportion de ballast " plus grande sera la quantité de paille que l'on pourra faire absorber à la vache.

Exemple:

Nous nous fixons comme but de remplacer le son de blé par un mélange de pommes de terre séchées et de tourteau de soja. Les normes alimentaires sont :

\begin{tabular}{|c|c|c|c|}
\hline & \multicolumn{3}{|c|}{ Pour I $000 \mathrm{~g}$ d'aliment, on a : } \\
\hline & $\begin{array}{l}\text { Albumine } \\
\text { digestible } \\
\mathrm{g}\end{array}$ & $\begin{array}{c}\text { Valeur amidon } \\
\qquad \mathrm{g}\end{array}$ & $\begin{array}{c}\text { Ballast } \\
\mathrm{g}\end{array}$ \\
\hline 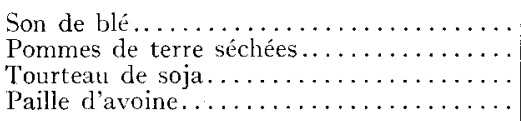 & $\begin{array}{r}91 \\
36 \\
+04 \\
10\end{array}$ & $\begin{array}{l}426 \\
737 \\
733 \\
170\end{array}$ & $\begin{array}{r}269 \\
90 \\
13 \\
408\end{array}$ \\
\hline
\end{tabular}

D'après le calcul des éléments fondamentaux nutritifs et d'après le vieux principe que deux aliments ou mélanges ont la même valeur nutritive quand ils possèdent la même quantité d'unités-amidon pour le même teneur en protéines digestibles, nous trouvons le résultat suivant:

\begin{tabular}{|c|c|c|c|}
\hline & $\begin{array}{c}\text { Albumine } \\
\text { digestible } \\
\mathrm{g}\end{array}$ & $\begin{array}{l}\text { Valeur amidon } \\
\mathrm{g}\end{array}$ & $\begin{array}{c}\text { Ballast } \\
\mathrm{g}\end{array}$ \\
\hline 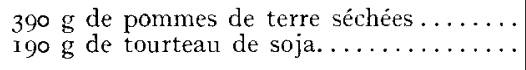 & $\begin{array}{l}14 \\
77\end{array}$ & $\begin{array}{l}287 \\
\text { I } 39\end{array}$ & $\begin{array}{r}35 \\
8\end{array}$ \\
\hline Total. & 9I & 426 & 43 \\
\hline
\end{tabular}

Ce mélange a donc la même richesse en protéine digestible et valeur amidon que le son de blé. Mais si nous considérons le ballast, le calcul se présente autrement : les deux aliments de remplacement sont pauvres en ballast et par conséquent permettent d'employer dans la ration de la paille d'avoine. En fait, l'équivalence réelle à tous les points de vue avec le son sera réalisée par le mélange suivant :

\begin{tabular}{|c|c|c|c|}
\hline & $\begin{array}{l}\text { Albumine } \\
\text { digestible } \\
\quad \mathrm{g}\end{array}$ & $\begin{array}{c}\text { Yaleur amidon } \\
\mathrm{g}\end{array}$ & $\begin{array}{l}\text { Ballast } \\
\mathrm{g}\end{array}$ \\
\hline 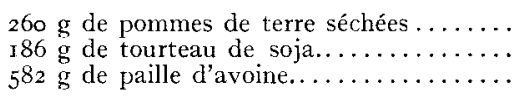 & $\begin{array}{r}9 \\
76 \\
6\end{array}$ & $\begin{array}{r}\text { I92 } \\
\text { I } 36 \\
98\end{array}$ & $\begin{array}{r}23 \\
8 \\
232\end{array}$ \\
\hline Total............ & 9 I & 426 & 263 \\
\hline
\end{tabular}


La question du ballast apparaît ici sous un jour essentiellement pratique, et ceci devient encore plus évident quand on considère le prix des aliments. Il est du reste à noter qu'il ne s'agit pas d'un calcul de rentabilité mais d'un exemple. Au début de I937 les prix des alịments cités plus haut, étaient les suivants en Allemagne centrale pour Ioo $\mathrm{kg}$ :

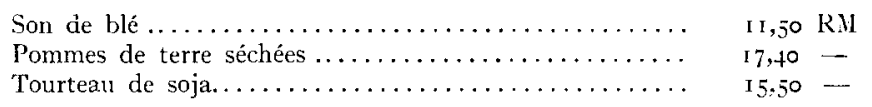

Le mélange de $39,0 \mathrm{~kg}$ de pommes de terre séchées et $19,0 \mathrm{~kg}$ de tourteau de soja qui, d'après le premier exemple équivaut à roo $\mathrm{kg}$ de son revient à 9,72 RM. Mais si, conformément à la théorie du ballast, on ajoute à ce premier mélange de la paille d'avoine pour obtenir un mélange avec la même teneur en ballast que le son, nous obtenons le deuxième mélange avec $26,0 \mathrm{~kg}$ de pommes de terre séchées, I8,6 kg de soja et 58,2 $\mathrm{kg}$ de paille d'avoine. Si nous supposons que cette paille est gratuite, le mélange coûte 7,32 RM. Nous voyons donc que ce deuxième mélange représente par rapport au premier une économie de 2,4 I RM.

La notion de valeur amidon se déduit de deux sources : $\mathrm{I}^{0}$ à partir des éléments nutritifs digestibles ; $2^{\circ}$ à partir du coefficient d'utilisation (WERTIGKEIT) résultant des recherches de KELLNER et calculé ensuite par lui pour les différents aliments au moyen d'un calcul global.

Ceci nous a amené à essayer de faire nos calculs en négligeant ce coefficient d'utilisation (WERTIGKEI'T) et en nous basant uniquement sur les éléments nutritifs digestibles, comme cela était l'usage avant l'époque de KELLNER.

KELLNER juge très défavorablement la paille, parce que c'est un aliment riche en fibres et avec un faible coefficient d'utilisation (WERTIGKEIT). Mais, nous venons de voir dans l'exemple ci-dessus que c'est seulement en introduisant de la paille dans la ration que nous réalisons un mélange entièrement comparable au son. Ceci nous amène donc à envisager nos calculs avec un autre point de vue. Nous déduisons des tables de KELINER les chiffres suivants :

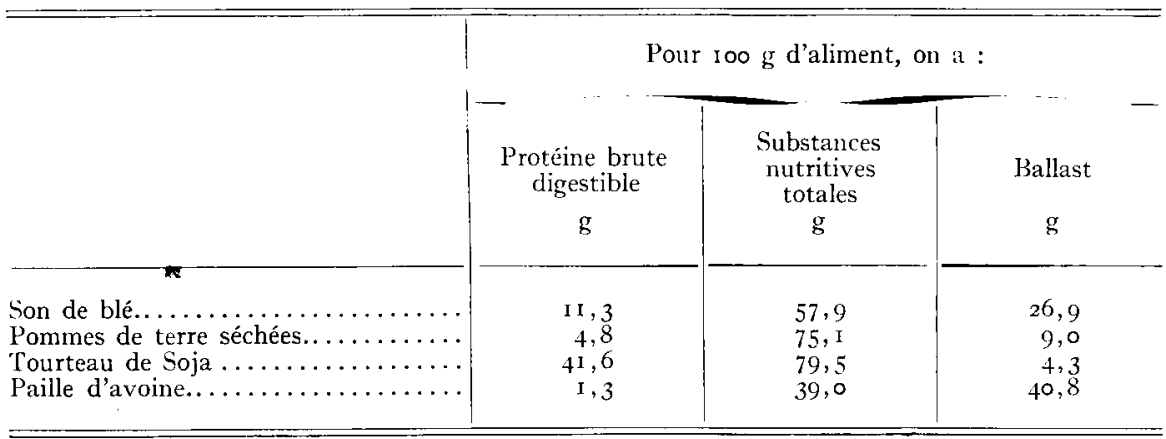


Si nous utilisons ces chiffres, l'équivalence du son est donnée par un mélange de :

$19,9 \mathrm{~kg}$ pommes de terre sèches

$23,0 \mathrm{~kg}$ de tourteau de soja

$63,0 \mathrm{~kg}$ de paille d'avoine.

Si nous nous basons sur les prix indiqués ci-dessous, en considérant la paille comme gratuite, ce mélange coûte $7,02 \mathrm{R}$. M. L'économie réalisée est de 2,70 R. M. Nos chiffres concordent bien avec ceux de KELLNER.

Nous pouvons donc réaliser nos calculs sans faire de rectification tenant compte du coefficient d'utilisation (Wertigkeit), mais en supposant qu'il sera possible pratiquement à l'agriculteur de faire absorber au ruminant la quantité maxima de paille et que, dans la réalité, le principe suivant soit valable : si l'animal est saturé en ballast d'une manière constante et totale, on peut, sans correction tenant compte du coefficient d'utilisation (Wertigkeit), calculer la valeur alimentaire d'un aliment à partir du total des éléments nutritifs, c'està-dire de la somme des éléments nutritifs digestibles.

Nous ne pousserons pas plus loin cette idée ; mais, elle nous amène à considérer le rapport entre le ballast et le coefficient d'utilisation (Wertigkeit).

\section{Ballast et coefficient d'utilisation (Wertigkeit)}

Les recherches fondamentales de KELL,NER sur le métabolisme des bovins à l'engrais amenèrent une surprise éclatante. Après qu'il eut déterminé la valeur de chacune des substances nutritives séparément, à savoir : albumine digestible, matière grasse, et fibres, en ce qui concerne leur efficacité sur l'engraissement des bœufs, il décida d'essayer les aliments eux-mêmes et découvrit un principe tout à fait nouveau qui peut s'exprimer comme suit :

Des aliments à haute digestibilité, c'est-à-dire avec un coefficient de digestibilité de $80-90 \%$ des substances organiques, avaient une action égale ou presque égale aux mélanges des substances nutritives pures. On pouvait dans ce cas, calculer le poids de viande et graisse (ou en calories équivalentes) pris par l'animal à partir des quantités digestibles d'albumine, matières grasses et hydrocarbones, à l'aide des chiffres standards déterminés par lui à la suite de ses recherches. Ces aliments sont donc totalement valorisés par l'animal. Mais, quand on utilise des aliments moins digestibles, c'est-à-dire dans lesquels le coefficient de digestibilité des substances organiques est inférieur à 70 , la quantité de viande et graisse calculée comme devant être prise par l'animal ne correspondait plus à celle réellement prise. Ce dernier chiffre était inférieur au chiffre théorique. Il y avait donc une chute de rendement dans la production. Ceci amena KELLNER à une nouvelle conception, celle du “ Coefficient d'utilisation "(Wertigkeit). Ce coefficient décroît d'autant plus que le coefficient de digestibilité est plus faible, comme le montre le tableau sommaire suivant: 


\begin{tabular}{|c|c|c|c|c|}
\hline & Blé & Son & Foin & Paille \\
\hline 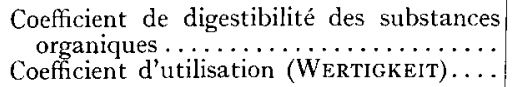 & $\begin{array}{l}86 \\
94\end{array}$ & $\begin{array}{l}7^{2} \\
78\end{array}$ & $\begin{array}{l}6 \mathbf{I} \\
69\end{array}$ & $\begin{array}{l}42 \\
24\end{array}$ \\
\hline
\end{tabular}

On peut représenter ces chiffres de manière inversée en indiquant au lieu de la proportion de substances organiques digestibles, la proportion non digestible, comparée à la chute de production. On a alors :

\begin{tabular}{l|c|c|c|c}
\hline & Blé & Son & Foin & Paille \\
\cline { 2 - 4 } & & & & \\
\hline Substances organiques non digestibles.... & 14 & 28 & 39 & 58 \\
Chute de production................. & 6 & 22 & $3^{\mathrm{I}}$ & $7^{6}$ \\
\hline
\end{tabular}

Or, la substance organique non digestible est le ballast. Nous en déduisons donc le principe suivant : la chute de production croît en même temps que la teneur en ballast. Il n'est pas question de proportionnalité entre les deux facteurs, mais ce parallélisme nous donne le droit de penser que nous avons trouvé là, la cause de ce phénomène inattendu qu'est la chute de production. KEI, LNER n'a pas suivi cette voie, mais a rendu les fibres responsables du phénomène. Il ne s'agissait pas pour lui de déterminer si les fibres telles qu'il les déterminait par l'analyse chimique étaient la cause de la chute de production. Il voulait surtout trouver un facteur mathématique, lui permettant de déterminer la valeur d'utilisation des nombreux aliments qu'il ne pouvait soumettre à des essais pratiques de métabolisme, de manière à calculer la quantité réelle de viande et graisse que ces aliments permettaient à l'animal de former. Il fut donc amené à définir la "correction pour teneur en fibres " conception qui n'est pas très heureuse.

Les discussions sur la partie fibreuse ont déjà une longue histoire. Les anciens chimistes analystes attachaient une grosse importance à la cellulose car ils la considéraient comme la partie non digestible des hydrocarbones. Ceci les amena à diviser les hydrocarbones en deux catégories : les fibres insolubles et non digestibles, et d'autre part, tout ce qui était soluble et par conséquent totalement digestible. HENNEBERG réussit cependant à démontrer qu'une partie des fibres contenues dans les fourrages et les pailles était digestible. Mais, par contre, trouva que l'extractif non azoté n'était jamais totalement digestible et en déduisit une relation compensatrice presque oubliée aujourd'hui qu'il appela "compensation " (" compensation " en français dans le texte).

L'extrait non azoté trouvé par analyse est la somme de l'extrait non azoté digestible et de la fibre digestible. On peut donc renverser le principe qui prend alors une forme plus pratique : le chiffre trouvé pour la fibre par analyse est la somme de l'extrait non azoté non digestible et de la fibre non digestible. 
Ce chiffre de la fibre trouvé par analyse représente dans la réalité (et en particulier avec les fourrages et les pailles) la plus grande partie de la substance organique non digestible. Pour savoir si on doit choisir comme chiffre de base le ballast ou la fibre de l'aliment, il faut d'abord connaître dans quelle mesure en dehors des hydrocarbones digestibles il y a lieu de tenir compte de la partie non digestible des deux autres groupes d'éléments nutritifs à savoir les protéines et les matières grasses. Si les chiffres pour les fibres et le ballast sont à peu près parallèles, il est évident que le chiffre pour le ballast est un peu plus élevé que le chiffre de fibres déterminé par analyse.

Mais naturellement on ne s'est pas contenté de ces considérations et on a essayé de déterminer de plus près les causes de cette étonnante chute de production. On y fut aidé par les observations que fit ZuNTZ dans ses recherches sur le métabolisme du cheval.

KELLNER avait d'abord déterminé la production due aux substances nutritives pures. Or, les substances nutritives pures se distinguent des aliments réels à deux points de vue :

$\mathrm{r}^{0}$ Les aliments réels doivent être broyés. Il y a donc à tenir compte du travail de mastication qui correspond à une dépense de substances nutritives.

$2^{\circ}$ Les aliments réels se distinguent des substances nutritives pures utilisées par KELLNER dans ses essais, en ce qu'ils ne sont pas entièrement digestibles, mais contiennent en même temps en quantités plus ou moins grandes des éléments non digestibles. Ces éléments sont le ballast. ZuNTZ avait déjà donné des coefficients de diminution de la valeur alimentaire analogues à ceux de KEILNER. Il avait alimenté un cheval avec du foin de prairie et avait déterminé combien de calories sont nécessaires pour le travail de digestion d'une part, et pour le travail de mastication d'autre part. Un $\mathrm{kg}$ de foin séché à l'air nécessitait pour le travail de mastication et son transport à travers l'intestin une dépense de 406 calories. On put déterminer par un essai direct que le travail de mastication absorbait 167 calories, de sorte qu'il restait 239 calories pour le transport du ballast à travers l'intestin. Si nous traduisons cela en unités-amidon, nous trouvons qu'un $\mathrm{kg}$ de foin exige la dépense de 70,8 unités-amidon pour le travail de mâchage. Comme le transport de 3 Io $\mathrm{g}$ environ de ballast à travers l'intestin représentait une dépense de 239 calories, on peut en déduire que le transport de $\mathrm{I} g$ de ballast exige $0,77 \mathrm{I}$ calories correspondant à la consommation de 0,327 unités-amidon.

Ces chiffres n'ont évidemment qu'une valeur relative, mais je les ai toujours utilisés dans mes cours pour mieux faire comprendre à mes élèves le phénomène de la chute de production. Ces chiffres ne pouvaient cependant permettre des conclusions définitives, car il aurait fallu alors avoir l'audace de supposer qu'on pouvait admettre que les conditions étaient les mêmes chez les ruminants que chez les chevaux. Toutefois, les deux chiffres de ZuNTZ m'amenèrent à dresser une table qui montre l'influence du ballast sur le coefficient d'utilisation (Wertigkeit), valeur qui reflète la chute de production. Il est du 
reste à remarquer que les chiffres obtenus par moi étaient en bonne concordance avec les chiffres calculés par KELLNER et qu'on trouve dans ses tables.

Pour simplifier, nous avons supposé que dans le cas des aliments séchés à 1'air libre, l'eau et les cendres représeentent un total de $20 \%$, c'est-à-dire que les substances organiques représentent $80 \%$ du poids de l'aliment. Donc, si nous supposons que ces substances organiques ont une digestibilité de Ioo $\%$, la quantité totale de substances nutritives est de $80 \%$, en admettant pour simplifier que la quantité de protéines digestibles est égale à la quantité d'hydrocarbones digestibles (la petite quantité de matière grasse étant inclue dans le chiffre d'hydrocarbones).

\section{Tableau IX}

Influence du ballast sur la valeur amidon demeurant disponible pour la production

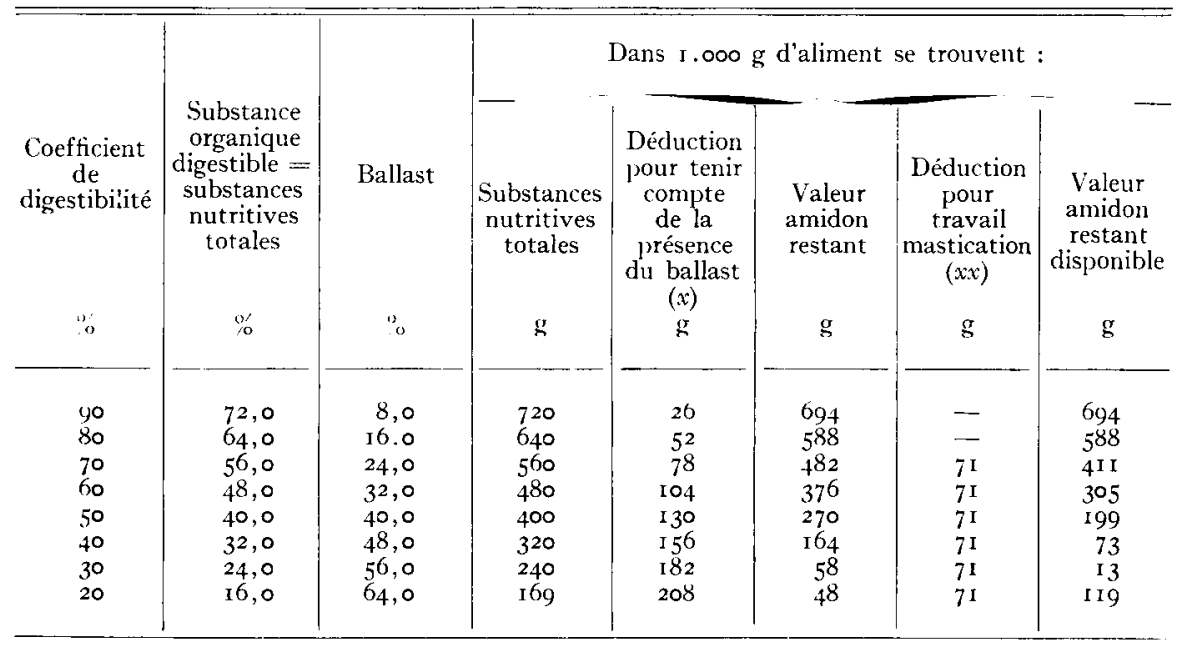

(x) par gramme de ballast, il est retranché 0,327 unités amidon.

$(x x)$ par $\mathrm{I}, 000 \mathrm{~g}$ d'aliment, il est retranché $7 \mathrm{I}$ unités amidon.

Le tableau IX indique les teneurs en unités-amidon et ballast au fur et à mesure que le coefficient de digestibilité décroît. On retranche des substances nutritives totales la quantité d'unités-amidon correspondant au travail de transport dans l'intestin, à savoir pour I g de ballast 0,327 unités-amidon.

Nous considérons comme aliments très digestibles ceux qui ont un coefficient de digestibilité de 90 et $80 \%$, lesquels n'exigent aucun travail sensible de mastication. A partir d'une digestibilité de $70 \%$ nous trouvons des aliments tels que le son et les fourrages grossiers et volumineux qui, en plus du travail de transport dans l'intestin exigent un travail de mastication. D'après ce que nous avons indiqué plus haut, nous estimons que le travail de mastication de $\mathrm{r}$ ooo $\mathrm{g}$ d'aliments correspond à une dépense standard de 7 I g d'unités-amidon. Ce dernier chiffre est retranché des unités amidon restant après soustraction 
de celles correspondant au transport dans l'intestin. Finalement, il reste les unités-amidon disponibles pour l'entretien et la production. Il est à remarquer que les aliments avec une digestibilité de $20 \%$ montrent déjà un déficit de 4,8 unités-amidon avant même qu'on ait déduit les unités nécessaires pour le tra-

TABleaU $\mathrm{X}$

\begin{tabular}{|c|c|c|}
\hline $\begin{array}{c}\text { Coefficient } \\
\text { de digestibilité }\end{array}$ & $\begin{array}{c}\text { Coefficient } \\
\text { d'utilisation } \\
\text { sans soustraction } \\
\text { du travail } \\
\text { de mastication }\end{array}$ & $\begin{array}{c}\text { Coefficient } \\
\text { d'utilisation } \\
\text { après soustriction } \\
\text { du travail } \\
\text { de mastication }\end{array}$ \\
\hline 90 & 96 & - \\
\hline 80 & 92 & - \\
\hline $\begin{array}{l}70 \\
6 \mathrm{c}\end{array}$ & $\begin{array}{l}86 \\
78\end{array}$ & $\begin{array}{l}73 \\
52\end{array}$ \\
\hline 50 & 67 & 40 \\
\hline 40 & 51 & 29 \\
\hline 30 & 24 & - \\
\hline 20 & - & - \\
\hline
\end{tabular}

vail de mastication. Avec les aliments possédant $30 \%$ de digestibilité, le déficit n'apparaît qu'après soustraction des unités-amidon, correspondant au travail de mastication. Le danger d'utiliser des aliments avec un coefficient de digestibilité aussi faible est illustré par ces chiffres de façon frappante.

Le tableau IX nous fournit une base pour examiner de plus près le coefficient d'utilisation (Wertigkeit). Ce coefficient se calcule à partir des substances nutritives totales et de la valeur amidon, restant après les soustractions ; c'est le pourcentage des valeurs amidon restant disponibles par rapport aux valeurs amidon primitivement fournies avec l'aliment. Le tableau $\mathrm{X}$ indique ces coefficients d'utilisation déduits du tableau IX.

\section{Tableau XI}

Caractéristiques de trois fourrages d'après les tables de Kellner

\begin{tabular}{|c|c|c|c|c|c|}
\hline Fourragə & $\begin{array}{c}\text { Protéïnes } \\
\text { digestibles }\end{array}$ & $\begin{array}{l}\text { Matière } \\
\text { grasse }\end{array}$ & $\begin{array}{l}\text { Extractif } \\
\text { non azoté }\end{array}$ & Fibres & $\begin{array}{l}\text { Substances } \\
\text { organiques }\end{array}$ \\
\hline Mauvaise qualité $\left\{\begin{array}{l}\text { totale } \ldots \ldots \ldots \ldots \ldots \\
\text { digestible.......... }\end{array}\right.$ & $\begin{array}{l}7,5 \\
3,4\end{array}$ & $\begin{array}{l}1,5 \\
c, 5\end{array}$ & $\begin{array}{l}38,2 \\
19,3\end{array}$ & $\begin{array}{l}33,5 \\
15,6 \\
\text { Ballast }\end{array}$ & $\begin{array}{r}80,7 \\
38,8 \\
=41,9\end{array}$ \\
\hline 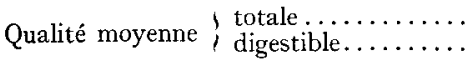 & $\begin{array}{l}9,7 \\
5,4\end{array}$ & $\begin{array}{l}2,5 \\
1,0\end{array}$ & $\begin{array}{l}4 \mathrm{I}, 4 \\
25,7\end{array}$ & $\begin{array}{l}26,3 \\
\text { I5,o } \\
\text { Ballast }\end{array}$ & $\begin{array}{r}79,9 \\
47,1 \\
=\quad 32.8\end{array}$ \\
\hline Très bonne qualité, totale $\ldots \ldots \ldots \ldots$ & $\begin{array}{r}\text { I } 3,5 \\
9,2\end{array}$ & $\begin{array}{l}3,0 \\
1,5\end{array}$ & $\begin{array}{l}40,4 \\
30,1\end{array}$ & $\begin{array}{l}19,3 \\
12,7 \\
\text { Ballast }\end{array}$ & $\begin{array}{r}76,2 \\
53,5 \\
=\quad 3^{2}, 7\end{array}$ \\
\hline
\end{tabular}

On voit nettement apparaître l'influence des soustractions. Les chiffres que $\mathrm{j}$ 'ai trouvés ainsi concordent bien avec ceux des tables de KELL,NER et cette 
concordance fut encore prouvée d'une autre manière. Dans les tables de KEILNER, je pris comme exemple trois espèces de fourrage. Je calculais le ballast à partir des substances organiques totales et $j$ 'en retranchais les substances organiques digestibles. Ces chiffres sont portés dans le tableau XI.

Des substances organiques digestibles, nous déduisons suivant la méthode connue de calcul, les substances nutritives totales, c'est-à-dire les subs-

Tableau XII

Comparaison des valeurs amidon Lehmann et Kellner

\begin{tabular}{|c|c|c|c|c|c|c|}
\hline \multirow[b]{2}{*}{ Fourrage } & \multirow{2}{*}{$\begin{array}{c}\text { Substances } \\
\text { nutritives } \\
\text { totales } \\
\text { (unités } \\
\text { amidon } \\
\text { brutes) }\end{array}$} & \multicolumn{3}{|c|}{ Soustraction d'unités amidon pour: } & \multicolumn{2}{|c|}{ Unités amidon réelles : } \\
\hline & & $\begin{array}{l}\text { Transport } \\
\text { dans } \\
\text { l'intestin }\end{array}$ & $\begin{array}{c}\text { Travail } \\
\text { de } \\
\text { mastication }\end{array}$ & $\begin{array}{c}\text { Total } \\
\stackrel{a}{a} \\
\text { soustraire }\end{array}$ & $\begin{array}{l}\text { LEHMANN } \\
\text { (calcul) }\end{array}$ & $\begin{array}{c}\text { KELLLNER } \\
\text { (tables) }\end{array}$ \\
\hline Mauvaist qualité. & 39,5 & I3,7 & 7,1 & 20,8 & 18,7 & 18,9 \\
\hline Qualité moyenne........ & 48,4 & 10,7 & 7,1 & I 7,8 & 30,6 & $3 \mathrm{I}, \mathrm{O}$ \\
\hline Très bonne qualité ....... & 55,5 & 7,4 & $7, \mathrm{I}$ & 14,8 & 41,0 & 40,6 \\
\hline
\end{tabular}

tances nutritives ayant une valorisation complète, c'est-à-dire un coefficient d'utilisation égal à roo. Puis, on soustrait 0,327 unités-amidon par gramme de ballast pour le transport dans l'intestin et on retranche, en outre, 7, I unitésamidon pour le travail de mastication par $\mathrm{I}$ ooo $\mathrm{g}$ d'aliment. On aboutit ainsi au tableau XII déduit du tableau XI.

\section{TABLEAU XIII}

Chute de production en fonction de la teneur en fibres et de la richesse en ballast

\begin{tabular}{|c|c|c|c|}
\hline & \multicolumn{3}{|c|}{ Pour $100 \mathrm{~g}$ de matières sèches, on a : } \\
\hline & $\begin{array}{c}\text { Chute } \\
\text { de production } \\
\text { (calories) }\end{array}$ & $\begin{array}{c}\text { Fibres } \\
\mathrm{g}\end{array}$ & $\begin{array}{c}\text { Ballast } \\
\mathrm{g}\end{array}$ \\
\hline Paille de blé, type a................. & 78,8 & 46,6 & 52,0 \\
\hline Paille de tlé, type b............... & 55,4 & 45,4 & $6 \mathrm{I}, 3$ \\
\hline Paille d'avoine...$\ldots \ldots \ldots \ldots \ldots \ldots \ldots$ & 40,8 & 39,0 & 50,9 \\
\hline Paille d'orge..$\ldots \ldots \ldots \ldots \ldots \ldots \ldots$ & $3^{6,4}$ & 35,7 & 46,9 \\
\hline Foin de prairie, type a.............. & 45,7 & 32,9 & 42,2 \\
\hline Foin de prairie, type $b . . \ldots \ldots \ldots \ldots$. & 44,7 & 30,9 & 31,2 \\
\hline 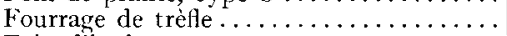 & 37,2 & 29,7 & 42,7 \\
\hline 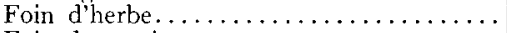 & 45,7 & $29, \mathrm{I}$ & 41,4 \\
\hline Foin de regain $\ldots \ldots \ldots \ldots \ldots \ldots \ldots$ & $\mathcal{H}^{2}, 9$ & 28,0 & $45, \mathrm{I}$ \\
\hline Moyenne.... & 47,5 & 35,3 & 46,0 \\
\hline
\end{tabular}

Les valeurs que j'ai calculées correspondent bien avec celles de KELLNER, et il paraît inutile de faire d'autres calculs. Il nous semble par contre utile de 
parler de 1'utilisation des chiffres standards que nous avons utilisés. Quoique je ne les ai utilisés que dans mes cours et ne les ai jamais publiés, ces chiffres me semblent avoir été utilisés par d'autres auteurs.

KELINER a examiné les rapports ci-dessus dans le cas de 7 aliments. Le résultat est donné dans son ouvrage, où il étudie la chute de production en fonction de la teneur en fibres et calcule ainsi la chute de production pour I $g$ de fibres, dans le tableau XIII.

J'ai indiqué également le ballast tel que j'ai pu le calculer à partir des données, assez vagues du reste, des tableaux de KELLNER.

Nous voyons, d'après ce tableau, que la chute de production est, en moyenne de $\mathrm{I}, 36$ calories pour $\mathrm{I} g$ de fibres ou $\mathrm{I}, 03$ calories pour I $\mathrm{g}$ de ballast.

Mais, examinons d'autres aliments contenant moins de fibres et de ballast que ceux du tableau ci-dessus et qui cependant causent une chute de production importante. Nous trouvons :

\section{TABLEAU XIII $b i s$}

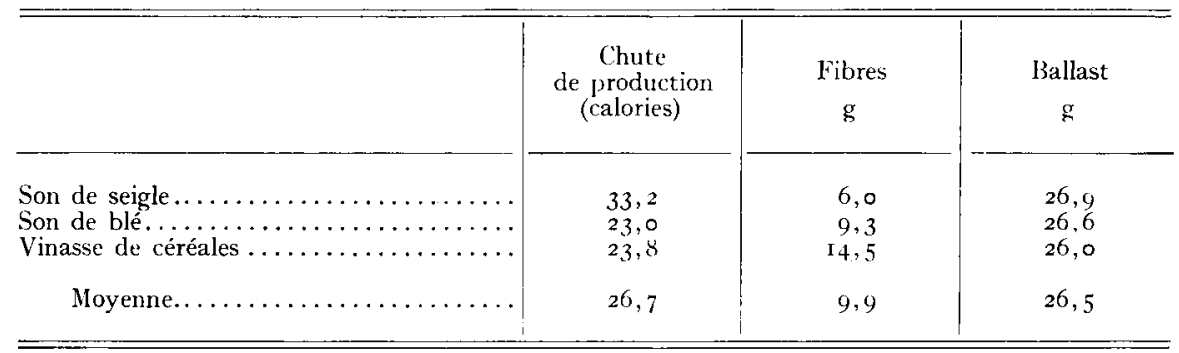

Pour ces trois aliments, nous trouvons donc une chute de production de 2,70 calories pour I $g$ de fibres et de I, or calories pour I $g$ de ballast.

Voyons maintenant les chiffres avec des moutures, nous avons :

TABLEAU XIII ter

\begin{tabular}{|c|c|c|c|}
\hline & $\begin{array}{l}\text { Chute } \\
\text { de production } \\
\text { (calories) }\end{array}$ & Fibres & $\begin{array}{c}\text { Ballast } \\
\mathrm{g}\end{array}$ \\
\hline $\begin{array}{l}\text { Mouture de seigle.................. } \\
\text { Mouture de fèverolles. } \ldots \ldots \ldots \ldots \ldots \ldots\end{array}$ & $\begin{array}{r}\mathrm{I} \mathrm{I}, \mathrm{I} \\
0,2\end{array}$ & $\begin{array}{l}2,2 \\
8,3\end{array}$ & $\begin{array}{r}9.6 \\
12,9\end{array}$ \\
\hline Moyenne................ & 10,2 & 5.3 & I I, 3 \\
\hline
\end{tabular}

I a chute de production est de I,93 calories pour I g de fibres et de 0,9I calories pour I $\mathrm{g}$ de ballast.

Comparons maintenant ces moyennes pour les trois espèces d'aliments considérés. Nous allons pouvoir mieux voir quelle méthode de calcul paraît la plus certaine. Nous trouvons : 


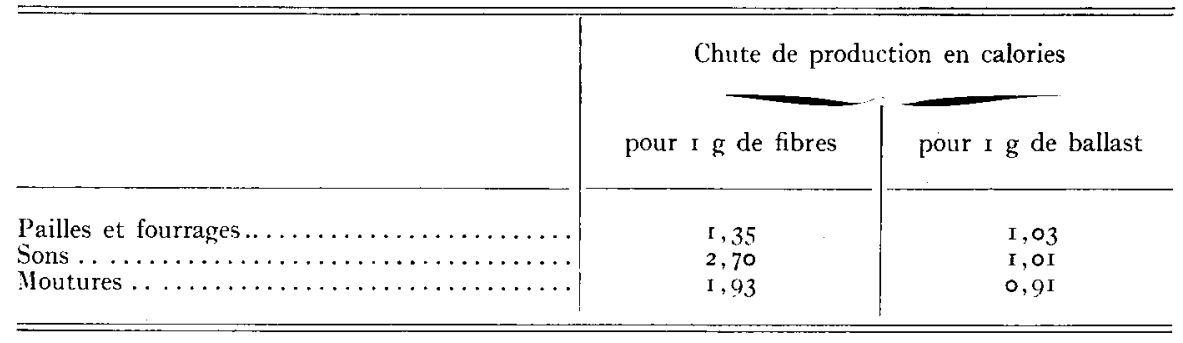

Les différents chiffres pour le ballast concordent très correctement, alors que ce n'est pas le cas avec les chiffres pour les fibres. Nous devons donc considérer que la comparaison est très en faveur du ballast comme base de calcul de la chute de production, autrement dit pour la méthode qui, a priori, apparaît du reste la plus naturelle.

Cette comparaison montre en outre, que la "correction pour fibres" indiquée par KeLLNER, comme étant de I,36 calories, soit ( 0,58 unités-amidon) ne peut être d'une application générale. Il avait déduit ce chiffre de ses recherches avec les pailles et fourrages (tableau XIII) et par conséquent il ne peut être utilisé que pour cette espèce d'aliments. D'après les tables que j'ai à ma disposition, je calcule qu'en moyenne, pour ces aliments, le rapport :

$$
\frac{\text { Fibres }}{\text { ballast }}=\frac{\text { IOO }}{\text { II } 7} \text {. }
$$

Donc, si KELLNER a trouvé pour I $g$ de fibres, une correction de 0,58 unitésamidon, on devrait en déduire que pour I $\mathrm{g}$ de ballast, la correction est de 0,50 unités-amidon. Ce chiffre, comme le montrent les petits tableaux XIII bis et ter, serait encore utilisable pour d'autres aliments que les pailles et les fourrages. Mais, si on voulait procéder à un tel calcul généralisé, les chiffres de ballast que nous avons indiqués ne pourraient être utilisés, car ils sont déduits des tables de KELLNER dont les données concernant le ballast sont très incertaines. Il serait nécessaire de calculer le ballast d'après des données statistiques plus sûres.

Mais le but de cette étude n'est pas de calculer une nouvelle correction pour les fibres. Cette correction ne peut, du reste, être un chiffre unique et constant, car il y a deux causes de la chute de production : le travail de mastication et le travail des intestins. KELLNER lui-même a du reste entrepris des recherches suivant une méthode qui est beaucoup plus sûre que celle de ZunTZ. Malheureusement, nous ne possédons pas les chiffres détaillés trouvés par lui et ne connaissons que le résultat final tel qu'il est indiqué par les chiffres de ses tables.

Il utilisa trois espèces de paille, détermina la chute de production pour Ioo $\mathrm{g}$ de matière sèche ; puis refit les mêmes essais avec ces trois espèces de paille broyées finement au moyen d'un malaxeur de sorte que dans ce dernier cas, l'animal n'avait pas à dépenser de travail de mastication : il étudia égale- 
ment la chute de production qui était due au travail de l'intestin. La différence trouvée entre les deux séries d'essais indiquait l'influence du travail de mastication.

Malheureusement, les possibilités de comparaison sont réduites par le fait que dans le cas de la paille non broyée, KelLnER utilisa deux fois de la paille de blé, et qu'avec la paille broyée, $\mathrm{jl}$ utilisa deux fois de la paille d'avoine. Ceci confirme combien la technique de nos recherches devrait être toujours d'une précision sévère. J'ai essayé de rectifier en partie cette erreur de méthode en utilisant des chiffres moyens et j'indique le résultat de mes calculs dans le tableau XIV:

\section{TABLEAU XIV}

Chute de production avec et sans travail de mastication

\begin{tabular}{|c|c|c|c|c|c|}
\hline & \multicolumn{3}{|c|}{ Pour $100 \mathrm{~g}$ de matière sèche } & \multicolumn{2}{|c|}{ Chute de production pour } \\
\hline & $\begin{array}{l}\text { Chute de } \\
\text { production } \\
\text { calories }\end{array}$ & $\begin{array}{c}\text { Fibres } \\
\mathrm{g}\end{array}$ & $\begin{array}{c}\text { Ballast } \\
\mathrm{g}\end{array}$ & $\begin{array}{l}\text { I } \mathrm{g} \text { de fibre } \\
\text { calories }\end{array}$ & $\begin{array}{c}\text { I g de ballast } \\
\text { calories }\end{array}$ \\
\hline $\begin{array}{l}\text { A) Avec travail de mastication : } \\
\text { Paille de blé.............. } \\
\text { Paille d'avoine } \ldots \ldots \ldots \ldots \ldots \ldots \\
\text { Paille d'orge } \ldots \ldots \ldots \ldots \ldots\end{array}$ & $\begin{array}{l}67,1 \\
40,8 \\
36,4\end{array}$ & $\begin{array}{l}46,0 \\
39,0 \\
35,7\end{array}$ & $\begin{array}{l}56,0 \\
50,9 \\
46,9\end{array}$ & $\begin{array}{l}1,46 \\
1,05 \\
1,02\end{array}$ & $\begin{array}{l}1,20 \\
0,80 \\
0,78\end{array}$ \\
\hline 13) $\begin{array}{l}\text { Sans travail de mastication : } \\
\text { Paille de blé } \ldots \ldots \ldots \ldots \ldots \ldots \\
\text { Paille d'avoine } \ldots \ldots \ldots \ldots \ldots \\
\text { Paille d'orge } \ldots \ldots \ldots \ldots \ldots\end{array}$ & $\begin{array}{l}31,4 \\
29,1 \\
28,6\end{array}$ & $\begin{array}{l}4^{2}, 2 \\
39,9 \\
+1,1\end{array}$ & $\begin{array}{l}56,0 \\
50,9 \\
46,9\end{array}$ & $\begin{array}{l}0,75 \\
0,73 \\
0,70\end{array}$ & $\begin{array}{l}0,56 \\
0,5^{8} \\
0,61\end{array}$ \\
\hline $\begin{array}{l}\text { C) Moyenne : } \\
\text { Avec travail de mastication. } \\
\text { Sans travail de mastication. }\end{array}$ & $\begin{array}{l}48,1 \\
29,7\end{array}$ & $\begin{array}{l}40,2 \\
4 I, I\end{array}$ & $\begin{array}{l}5 \mathrm{I}, 3 \\
5 \mathrm{I}, 3\end{array}$ & $\begin{array}{l}1,20 \\
0,73\end{array}$ & $\begin{array}{l}0,94 \\
0,5^{8}\end{array}$ \\
\hline
\end{tabular}

Les trois espèces de paille diffèrent beaucoup au point de vue de la teneur en fibres. Le résultat ne peut être atteint qu'avec les chiffres des moyennes et il est nécessaire de faire un léger calcul correctif pour que la teneur en fibres soit exactement la même dans les expériences avec et sans travail de mastication. Nous avons alors les moyennes suivantes:

\begin{tabular}{|c|c|c|c|c|c|}
\hline & \multicolumn{3}{|c|}{ Pour roo g de małière sèche } & \multicolumn{2}{|c|}{ Chute de production pour } \\
\hline & $\begin{array}{l}\text { Chute de } \\
\text { production } \\
\text { calories }\end{array}$ & $\begin{array}{l}\text { Fibres } \\
\qquad \mathrm{g}\end{array}$ & $\begin{array}{l}\text { Ballast } \\
\qquad g\end{array}$ & I g de fibre & I g de ballast \\
\hline $\begin{array}{l}\quad \text { Moyenne : } \\
\text { Avec travail de mastication.... } \\
\text { Sans travail de mastication..... }\end{array}$ & $\begin{array}{l}48,1 \\
29, I\end{array}$ & $\begin{array}{l}40,2 \\
40,2\end{array}$ & $\begin{array}{l}5 \mathrm{I}, 3 \\
5^{\mathrm{I}}, 3\end{array}$ & $\begin{array}{l}\mathrm{I}, 20 \\
0,73\end{array}$ & $\begin{array}{l}0,94 \\
0,5^{8}\end{array}$ \\
\hline
\end{tabular}


Le travail de mastication et le travail de l'intestin additionnés ont donc causé une chute de production de 48 , I calories pour roo $\mathrm{g}$ de matière sèche. La chute de production pour les mêmes espèces de paille avec la même teneur en fibres a été sans travail de mastication de 29,0 calories pour roo g de matière sèche. On peut donc en déduire que le travail de mastication a exigé la dépense de r9, I calories (équivalentes à 8, Io unités-amidon) pour Ioo g de substance sèche. Si nous estimons que ces pailles contenaient $86 \%$ de matière sèche, le travail de mastication a exigé la dépense de 6,97 unités-amidon pour Ioo $\mathrm{g}$ d'aliment. La deuxième série d'essais montre que le travail des intestins exige 0,73 calories (équivalentes à $0,3 \mathrm{I}$ unités-amidon) pour I g de fibres, ou 0,58 calories (équivalentes à 0,246 unités-amidon) pour I $g$ de ballast.

Mais il est possible d'utiliser d'une autre manière les résultats de ces recherches : on peut en effet pour les différentes espèces de paille, revenir en arrière, et soustraire de la chute de production, le travail des intestins, ce qui nous donne le travail de mastication. Bien entendu, nous devons tenir compte des différences de teneurs en fibres. Le tableau XV où tous les chiffres indiqués sont des calories (sauf pour les fibres) indique le résultat du calcul fait de cette manière :

\section{Tableau XV}

\begin{tabular}{|c|c|c|c|c|c|}
\hline & \multicolumn{5}{|c|}{ Pour roo g de matière sèche, on a : } \\
\hline & $\begin{array}{c}\text { Chute } \\
\text { de } \\
\text { production } \\
\text { cal }\end{array}$ & $\begin{array}{c}\text { Fibres } \\
g \\
g\end{array}$ & $\begin{array}{c}\text { Travail de } \\
\text { l'intestin } \\
\text { pour r g } \\
\text { de fibres } \\
\text { cal }\end{array}$ & $\begin{array}{l}\text { Travail } \\
\text { de } \\
\text { l'intestin } \\
\text { cal }\end{array}$ & $\begin{array}{c}\text { Travail } \\
\text { de } \\
\text { mastication } \\
\text { cal }\end{array}$ \\
\hline Paille de blé & $67, \mathrm{I}$ & 46,0 & 0,75 & 34,5 & 32,6 \\
\hline Paille d'avoine ................ & 40,8 & 39,0 & 0,73 & 28,4 & $\mathrm{I} 2,4$ \\
\hline Paille d'orge $\ldots \ldots \ldots \ldots \ldots \ldots \ldots$ & 36,4 & 35,7 & 0,70 & 24,9 & II, 5 \\
\hline 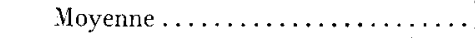 & $48, \mathrm{I}$ & 40,2 & 0,73 & 29,3 & 18,8 \\
\hline
\end{tabular}

Dans ces trois exemples, le travail de mastication se révèle comme le seul des facteurs, déterminés d'une manière exacte, qui n'est pas une grandeur constante. Exprimé en unités-amidon, le travail de mastication pour roo $\mathrm{g}$ de matière sèche, est de :

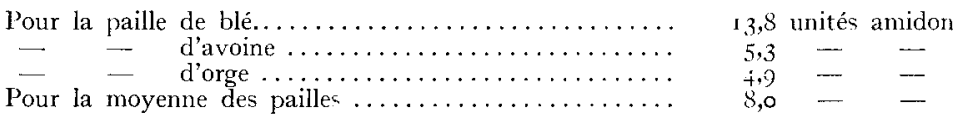

La moyenne à soustraire pour le travail de mastication est donc de 6,9 unités-amidon, (soit $8 \times 0,86$ ) pour roo g d'aliment.

Le résultat important montré par ces recherches exactes est donc la grande différence entre la paille de céréales d'hiver, et la paille de céréales d'été. 
On peut de manière analogue, quoiqu'avec moins d'exactitude, calculer le travail de mastication pour différentes sortes de fourrages, que KELLNER a étudiés dans ses expériences. Mon calcul m'a amené pourr trois espèces de fourrage, à la valeur moyenne de 9,4 unités-amidon pour roo $\mathrm{g}$ de matière sèche et à 8,0 unités-amidon pour Ioo $\mathrm{g}$ d'aliment (à $86 \%$ de matière sèche). Ce dernier chiffre me paraît être celui en moyenne le plus près de la réalité, dans l'hypothèse où on estime que 1 'on peut fixer un chiffre moyen pour le travail de mastication. Dans ce qui va suivre, je vais utiliser ce chiffre moyen.

Le progrès fondamental que KELLNER a fait réaliser dans la connaissance des aliments fourragers est la nouvelle conception qu'il a traduite, par son "coefficient d'utilisation" (Wertigkeit). Il est probable, comme il ressort de ma présente étude, que ce " coefficient d'utilisation " est influencé par la teneur en ballast et qu'il diminue quand le coefficient de digestibilité de l'aliment diminue. Si, reprenant une de nos bases simplifiées, nous admettons que dans les aliments séchés à l'air libre, le total de l'eau et des cendres représente $20 \%$ et que contrairement à notre calcul provisoire précédent, nous admettons une chute de production de 0,246 unités-amidon par g de ballast, nous pouvons d'abord calculer le coefficient d'utilisation, sans travail de mastication ; puis, nous retrancherons pour ce travail de mastication, par roo $\mathrm{g}$ d'aliment, la valeur indiquée plus haut de 8 ,o unités-amidon. Nous obtenons alors le tableau XVI :

\section{TABLEAU XVI}

\section{Coefficient d'utilisation avec et sans travail de mastication}

\begin{tabular}{c|c|c}
$\begin{array}{c}\text { Coefficient } \\
\text { de digestibilité } \\
\text { des substances } \\
\text { organiques }\end{array}$ & $\begin{array}{c}\text { Coefficient } \\
\text { d'utilisation } \\
\text { sans travail } \\
\text { de mastication }\end{array}$ & $\begin{array}{c}\text { Coefficient } \\
\text { d'utilisation } \\
\text { avec travail } \\
\text { de mastication }\end{array}$ \\
\hline 90 & & \\
80 & 97 & 97 \\
70 & 94 & 82 \\
60 & 90 & 67 \\
50 & 75 & 56 \\
40 & 63 & 25 \\
30 & 46 & 9 \\
20 & 2 & -2 \\
\hline
\end{tabular}

Ce tableau va nous permettre d'aboutir à des conclusions importantes, dont du reste beaucoup ont été indiquées déjà par moi dans mes cours.

Le coefficient de digestibilité de 90 correspond à des aliments avec une digestibilité presque totale et par conséquent n'exige aucun travail de mastication. KELLNER nous en a apporté la confirmation avec ses essais sur les tourteaux et les pommes de terre.

Le coefficient de digestibilité de 80 est celui d'aliments chez lesquels on voit déjà apparaître une chute de production due au travail de mastication. 
Dans cette catégorie, nous trouvons les pulpes de betteraves qui, sèches ou humides, ont un coefficient de digestibilité de 77 à $80 \%$ et que KELLNER a étudiées dans ses recherches. Le coefficient d'utilisation (Wertigkeit) a été mesuré par KELLNER comme étant de 78 , et celui des pulpes fraîches, qui nécessitent aucun travail de mastication, a été trouvé par lui comme étant de 94. Il en résulte que dans la pratique, on ne devrait utiliser les pulpes séchées que broyées ou gonflées dans l'eau. Or, cette conséquence des travaux de KELLNER ne semble avoir été jusqu'ici que très peu prise en considération. La valeur alimentaire des pulpes séchées à l'air est de 5I,9 unités-amidon et s'élève si on supprime le travail de mastication à 62,6 unités-amidon, soit une augmentation de valeur alimentaire de $20,7 \%$.

Dans le groupe avec le coefficient de digestibilité 70 , nous trouvons les sons. Ceux-ci exigent un important travail de mastication. En effet, KELLNER a trouvé des coefficients d'utilisation (Wertigkeit) de 79 pour le son de seigle et 77 pour le son de blé.

Si on broyait les sons et supprimait ainsi le travail de mastication, le coefficient d'utilisation (Wertigkeit) des sons monterait à 9o. Ceci, exprimé en unités-amidon amènerait les améliorations suivantes de valeur alimentaire :

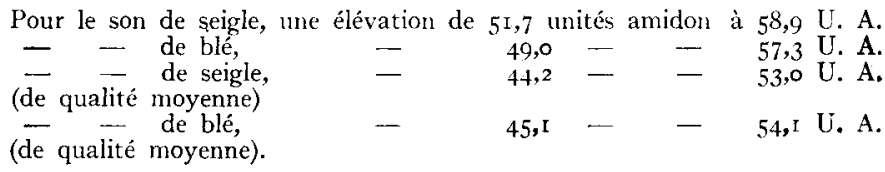

Nos tables ont malheureusement négligé cette différence entre les sons bruts et les sons broyés, du fait que KELINER et les savants qui suivirent avaient leurs vues obscurcies par la notion de "correction pour fibres ". De même, l'industrie a toujours négligé les possibilités qu'elle aurait pu offrir à l'agriculture en broyant les sons et s'est toujours contentée d'utiliser ses moulins pour faire des farines avec les grains entiers.

Les fourrages appartiennent à la catégorie avec le coefficient de digestibilité 60 . On sait que le travail de mastication est beaucoup plus faible avec la plante verte qu'avec le fourrage que l'on en tire. Les recherches de ZuNTZ avec le cheval l'ont bien démontré. Le phénomène est le même avec les ensilages de toute espèce, dont les caractéristiques sont quelquefois, mais rarement prises en considération dans nos tables.

Par contre, on ne parle jamais des farines de fourrage dont la valeur alimentaire est très augmentée par l'opération de broyage. Comme exemples, nous prendrons un foin de prairie et un fourrage de trèfle de valeur moyenne dont les coefficients de digestibilité sont respectivement de 60 et $6 \mathrm{I} \%$.

Ces deux fourrages ont, grâce au broyage, leur valeur alimentaire qui l'élève de 32,8 unités-amidon à 4I,6 unités-amidon.

L'importance économique de ce broyage est particulièrement sensible dans le cas des fourrages de première qualité. Je calcule dans ce cas les augmentations suivantes de valeur alimentaire : 
Foin de prairie I $^{\text {re }}$ qualité :

Élévation par broyage de 37,7 unités-amidon à 46,8 unités-amidon.

Fourrage de trèfle de $\mathrm{I}^{\mathrm{re}}$ qualité :

Elévation par broyage de 36,5 unités-amidon à 45,3 unités-amidon.

Or, nous avons vu que les sons de seigle et de blé avaient des valeurs alimentaires de 44,2 et $45, \mathrm{r}$ unités-amidon. Il en résulte donc que le broyage nous permet d'obtenir des farines de foin de prairie et de fourrage de légumineuses ayant la valeur alimentaire du son. La production de telles farines est non seulement justifiée, mais ne peut être que fortement recommandée dans les circonstances économiques actuelles. Malheureusement, les farines de foin et de fourrage que nous trouvons sur le marché depuis des dizaines d'années n'ont pas aidé à faire apprécier le broyage, du fait que l'on n'avait pas compris qu'il fallait surtout choisir pour le broyage des foins et fourrages de première qualité.

Les coefficients de digestibilité 50 et 40 sont ceux des différentes espèces de paille. Si on supprime pour les pailles le travail de mastication, la valeur alimentaire est doublée ; mais il s'agit de savoir si les dépenses pour broyer ces pailles se justifient économiquement. Le gonflement dans l'eau, comme avec les pulpes séchées, n'apporte guère d'économie. C'est seulement la désintégration chimique qui présente des avantages, et à deux points de vue : diminution de la teneur en ballast, et réduction du travail de mastication; c'est donc une méthode qui ouvre beaucoup de possibilités et mérite des recherches pour la mettre au point.

Les aliments dont le coefficient de digestibilité est de $30 \%$ ne méritent guère d'être employés et ne peuvent servir le cas échéant qu'à remplir l'estomac ou aider à fournir quelques calories à l'animal. Le travail de mastication et de l'intestin signifie dans ce cas une diminution de la production de graisse de l'animal, et ces aliments apportent seulement des calories pour réchauffer le corps de l'animal, dans la mesure où celui-ci a besoin de ces calories. EHRENBERG a déjà, il y a bien des années, attiré notre attention sur l'utilisation possible de mauvaises pailles pour nourrir en hiver des chevaux en état de sousalimentation. Mais cet avantage peut, dans le cas des ruminants recevant suffisamment de calories, se transformer facilement en désavantage.

\section{Notice bibliographique sur le Professeur-Docteur Franz LEHMANN}

(Tierernâhrung, Band I2, I940, pages 297-298)

Franz Lehmann, le maître vénéré de la Science de l'Alimentation, a fêté son quatrevingtième anniversaire le 28 avril 1940.

Notre revue, qui est consacrée à l'une des branches principales de l'activité du savant, ne peut laisser passer cet anniversaire sans adresser ses voux à l'illustre professeur, et sans rappeler sa brillante carrière de savant.

Fils d'un agriculteur, Lenmann naquit dans le Pays d'Anhalt. Il étudia les Sciences Naturelles à Iéna, Berlin et Göttingen. Il finit ses études le 27 février I 884 à Göttingen par 
un travail sur la chimie organique. Peu de temps après, il devint assistant de HENNEBERG, à la Station de Recherches Agronomiques de Göttingen et passa en I 889 à cette Université, sa thèse de Doctorat sur la valeur nutritive de la cellulose. Son maître HENNEBERg et l'Université de Göttingen ont eu une influence déterminante sur la carrière de LEHMANN.

En I $89 \mathrm{I}$, il succéda à Henneberg, comme Directeur de la Station de recherches, et fut nommé Professeur de Chimie Animale. Toutes les conditions étaient ainsi créées pour lui permettre de développer son activité de chercheur, et LEIIMANN se consacra avec ardeur à ce travail. La méthode qu'HENNEBERG avait créée pour l'étude de la physiologie du métabolisme reçut grâce à LEHMANN, sa forme améliorée définitive.

Grâce à cette méthode, LeHManN put entreprendre de vastes recherches sur la valeur nutritive de nombreux aliments. Mais LEHMANN ne se contenta pas d'étudier les aliments courants ; il considéra que sa tâche consistait surtout à trouver de nouveaux aliments. Il se consacra en particulier, à l'étude des aliments fournis par la culture de la betterave à sucre, et reconnut l'importance des queues de betteraves sucrières. Il fut le premier à suggérer le séchage de ces feuilles, et entreprit les premières recherches à ce sujet.

Il consacra d'autres travaux à la désagrégation chimique des pailles, méthode qui devait se révéler très importante lors de la première guerre mondiale.

Mais, ses travaux les plus importants concernèrent ses vastes recherches sur l'engraissement des cochons et des volailles, lesquelles sont maintenant universellement connues sous le nom de "Méthode de Göttingen ". Partant de points de vue tout à fait nouveaux, il réussit par cette méthode à combiner de la manière la plus parfaite, les connaissances théoriques de la science de l'Alimentation animale avec les nécessités de la pratique agricole. Ces méthodes se répandirent et furent appréciées bien au-delà des frontières de l'Allemagne.

Puis, le champ d'activité de Lehmann dépassa le cadre de l'Alimentation des animaux. Il s'intéressa à la question générale de la nutrition et en particulier à la "répartition des porteurs de substances nutritives " tels qu'il les fit apparaître dans sa conception de "l'Économie des substances nutritives ". Il fut l'un des premiers à entreprendre avec l'homme des essais sur l'utilisation des aliments, et obtint des données fondamentales sur l'utilisation des sons et du foin par l'homme et les animaux.

Aujourd'hui, à l'âge de 80 ans, LEHMANN grâce à son excellent état de santé, se tient en contact permanent avec toutes les questions concernant le domaine de ses activités scientifiques, et travaille continuellement à l'interprétation de ses propres recherches antérieures. 